
\title{
Research S Surate \\ Proton pump inhibitors may reduce the efficacy of ribociclib and palbociclib in metastatic breast cancer patients
}

KADIR ESER ( $\nabla$ drkadireser@gmail.com )

ARIF HAKAN ÖNDER

EMEL SEZER

TIMUÇiN ÇiL

ALI INAL

BANU ÖZTÜRK

VEHBI ERÇOLAK

BERNA BOZKURT DUMAN

HALIL ÇELIK

TOLGA KÖŞECI

OĞUZHAN KESEN

\section{Research Article}

Keywords: breast cancer, ribociclib, palbociclib, proton pump inhibitors, PFS

Posted Date: December 2nd, 2021

DOl: https://doi.org/10.21203/rs.3.rs-1131962/v1

License: (1) This work is licensed under a Creative Commons Attribution 4.0 International License. Read Full License 


\section{Abstract}

\section{Introduction}

Approximately $20-33 \%$ of all cancer patients are treated with acid reducing agents (ARAs), most commonly proton pump inhibitors (PPIs), to reduce gastro esophageal reflux disease symptoms. Palbociclib and ribociclib are weak base so their solubility depends on different $\mathrm{pH}$. The solubility of palbociclib dramatically decreases to $<0.5 \mathrm{mg} / \mathrm{ml}$ when $\mathrm{pH}$ is above 4,5 but ribociclibs' solubility decreases when $\mathrm{pH}$ increases above 6,5 . In the current study, we aimed to investigate the effects of concurrent PPIs on palbociclib and ribociclib efficacy in terms of progression free survival in metastatic breast cancer $(\mathrm{mBC})$ patients.

\section{Patients and methods}

We enrolled hormone receptor-positive, HER2-negative $\mathrm{mBC}$ patients treated with endocrine treatment (Letrozole or fulvestrant) combined palbociclib or ribociclib alone or with PPI accompanying our observational study. During palbociclib/ribociclib therapy, patients should be treated with "concurrent PPIs" defined as all or more than half of treatment with palbociclib/ribociclib, if no PPI was applied, it was defined as 'no concurrent PPI', those who used PPI but less than half were excluded from the study. All data collected from real life retrospectively.

\section{Results}

Our study included 217 patients, 105 of whom received palbociclib and 112 received ribociclib treatment. Of 105 patients who received Palbociclib, 65 were on concomitant PPI therapy, 40 were not. Of the 112 patients who received ribociclib, 61 were on concomitant PPI therapy, 51 were not. In the palbociclib group, the PFS of the patients using PPI was shorter than the PFS of the patients not using (13.04 months vs. unreachable, $p<0.0001)$. it was determined that taking PPI was an independent predictor of shortening PFS $(p<0.001)$ in the multivariate analysis, In the ribociclib group, the PFS of the patients using PPI was shorter than the PFS of the patients not using (12.64 months vs. unreachable, $p=0.003)$. It was determined that taking PPI was single statistically independent predictor of shortening PFS ( $p=0.003$, univariate analysis).

\section{Conclusions}

Our study demonstrated that concomitant usage of PPIs was associated with shorter PFS in mBC treated with both ribociclib and especially palbociclib. If it needs to be used, PPI selection should be made carefully and low-strength PPI or other ARAs (eg H2 antagonists, antacids) should be preferred.

\section{Introduction}

Targeted drugs such as thyrosine kinase inhibitors and cyclin-dependent kinase inhibitors are widely used in cancer patients. These drugs are oral medications, so, gastric $\mathrm{pH}$ has a significant effect on drug efficacy. There were many influencing factors on gastric $\mathrm{pH}$, such as feeding and concomitant medications. These drugs can be dissolved well when the appropriate $\mathrm{pH}$ is established. Approximately $20-33 \%$ of all cancer 
patients are treated with acid reducing agents (ARAs), most commonly proton pump inhibitors (PPIs), to reduce gastroesophageal reflux disease symptoms. PPIs also interact via the pharmacological and solubility pathways [1]. For this reason, drug-drug interactions (DDIs) at the time of absorption should be considered as one of the causes of treatment failure in cancer patients [2]. In fact, gastric $\mathrm{pH}$ elevation by PPIs reduces the oral bioavailability of many drugs used in cancer. This situation is demonstrated to be significant especially in those with exponentially decreasing solubility in the $\mathrm{pH}$ range 1-4. [3, 4]. The type of anticancer drugs determines the clinical occurrence of these changes [5]. It has been reported that long-term acid suppression by PPIs reduces the antitumor efficacy of pazopanib and capecitabine, while this effect of PPIs has not been found in clinical outcomes on patients treated with epidermal growth factor receptor (EGFR) thyrosine kinase inhibitors [6-8].

Ribociclib and palbociclib are oral cyclin-dependent kinase 4 and $6(C D K 4 / 6)$ inhibitors that arrest the cell cycle by inhibiting DNA synthesis inhibition[9]. The clinical efficacy of the combination of a CDK 4/6 inhibitor with aromatase inhibitors or fulvestrant has been acknowledged as the invention therapy in the first-second line treatment of human epidermal growth factor receptor 2 (HER2) negative hormone receptor positive premenopausal and postmenopausal women with advanced breast cancer [10-13]. Palbociclib is a weak base so its solubility depends on $\mathrm{pH}$. The solubility of palbociclib dramatically decreases to $<0.5 \mathrm{mg} / \mathrm{ml}$ when the $\mathrm{pH}$ is above 4.5 (i.e. gastric pH typically achieved by PPI). Ribociclib is also a weak base and its solubility decreases when the $\mathrm{pH}$ increases above 6.5 . Medicines are usually taken with $200-250 \mathrm{ml}$ of water. The in vitro solubility of ribociclib was investigated in biorelevant media consisting of simulated feeding (pH 5.0) and hungry ( $\mathrm{pH}$ 6.5) intestinal fluid. The maximum dose of ribociclib $(600 \mathrm{mg})$ was completely dissolved in $250 \mathrm{~mL}$ of biorelevant media [14]. Concomitant use of palbociclib with rabeprazole was found to reduce the mean area under the concentration time curve (AUC) - maximum plasma concentration (Cmax) 62\% - 80\% at fasting and $13 \%-41 \%$ at fed. [15]. Cinical trial data and population pharmacokinetics showed that ribociclib absorption was similar at various stomach $\mathrm{pH}$ values that occur after food intake or concomitant use of PPIs $[14,16]$. According to our knowledge to date, there are insufficient data on DDIs between palbociclib and PPIs other than rabeprazole.

In the current study, we aimed to investigate the effects of concurrent PPIs on palbociclib and ribociclib efficacy in terms of progression free survival in patients with estrogen-positive, HER2-negative metastatic breast cancer $(\mathrm{mBC})$ treated with palbociclib/ribociclib as a first line or subsequent line of treatment.

\section{Patients And Methods}

We enrolled hormone receptor-positive, HER2-negative $\mathrm{MBC}$ patients treated with palbociclib or ribociclib alone or with PPI accompanying our observational study. Tumors with estrogen receptors in patients with metastatic breast cancer if expression is $>10 \%$, we defined hormone receptor positive as HER2-negative as a score of 0 or $1+$ by immunohistochemistry and negative staining by SISH (silver in situ hybridization)/FISH (fluorescent in situ hybridization) in those with a score of $2+$ in immunohistochemistry. During palbociclib/ribociclib therapy, patients were treated with "concurrent PPIs" defined as all or more than half of treatment with palbociclib/ribociclib, If no PPI was applied, it was defined as 'no simultaneous PPI'. Those who used PPIs but less than half were excluded from the study. Based on previous endocrine time response, those with endocrine sensitivity (if relapsed at least 12 months after completion of adjuvant endocrine therapy or de novo metastatic 
breast cancer) or those who are endocrine resistant (relapse while receiving adjuvant therapy or recurrence within 12 months of discontinuation of adjuvant endocrine therapy) [17].

All clinicians in our study performed pharmacological and clinical interventions in real life according to clinical practice. One course of treatment was given as 28 days, consisting of 21 consecutive days of full and 7 days of blank treatment. Specifically, palbociclib orally at a dose of $125 \mathrm{mg}$, ribociclib orally at a dose of $600 \mathrm{mg} / 21$ days on and 7 days off), 28-day full cycle plus fulvestrant or letrozole were administrated. Ribociclib dose reduction was made to $400 \mathrm{mg}$, and palbociclib dose reduction was made to $100 \mathrm{mg}$ based on the toxicity profile. No lower dose was used in any patient. PPIs (lansoprazole $30 \mathrm{mg}$, esomeprazole $40 \mathrm{mg}$, omeprazole 40 $\mathrm{mg}$, pantoprazole $40 \mathrm{mg}$, rabeprazole $20 \mathrm{mg}$ dose) were recommended to take 30 minutes before breakfast. Ribociclib was used whether hungry or full, and palbociclib it was used with lunch. Strong inhibitors or inducers of cytochrome P450 3A4 (CYP3A4) while taking both drugs should be avoided. The doctors who wrote the prescription followed the patients' condition in accordance with the recommendations. Toxicity was assessed according to the World Health Organization (WHO) criteria classification. Approval was obtained from the local ethics committee in accordance with the Declaration of Helsinki, and the study was conducted accordingly.

\section{Statistical Analysis}

Eastern Cooperative Oncology Group (ECOG) performance status, hormone sensitivity, premenopausal versus postmenopausal status, treatment lines, visceral versus bone disease, and the number of tumor sites in absolute and median and relative frequencies and quantitative factors are categorical variables. The time from initiation of CDK combination therapy to progression was defined as PFS. For calculating PFS, generating survival curves and log-rank testing, the Kaplan-Meier method was used. Independent risk factors for PFS were determined with the Cox hazard regression method.

\section{Results}

Our study included 217 patients, 105 of whom received palbociclib and 112 of whom received ribociclib treatment. Of 105 patients who received palbociclib, 65 were on concominant PPI therapy, and 40 were not. Of the 112 patients who received ribociclib, 61 were on concominant PPI therapy, and 51 were not.

Forty-nine patients received, palbociclib combined with letrozole as first-line endocrine therapy (endocrine sensitive) and 56 endocrine refractory patients used fulvestrant as first- or subsequent line treatment. Of the patients treated with palbociclib, $49(46.7 \%)$ received the $125 \mathrm{mg}$ dose, and $56(53.3 \%)$ received the $100 \mathrm{mg}$ dose. Ribociclib was used as first line endocrine therapy (endocrine sensitive) with letrozol in 66 patients, and combined with fulvestrant as a first or subsequent line of therapy in 46 endocrine refractory patients. For patients treated with ribociclib, 51 (45.6\%) patients received the $600 \mathrm{mg}$ dose, and 61 (54.4\%) patients received the $400 \mathrm{mg}$ dose. There was no significant difference between the patients who took PPIs and those who did not in either the palbociclib group or the ribociclib group. The clinical characteristics for both drug groups separately are shown in Table 1.

In the palbociclib group, the PFS of the patients using PPIs was shorter than the PFS of the patients not using PPIs (13.04 months vs. unreachable, $p<0.0001$, respectively; Figure $1 \mathrm{~A})$. After a mean follow-up of 13 months, $83 \%$ of the patients who did not take PPIs did not progress. Univariate analysis included age, CDK combination, 
number of metastatic sites, ECOG, menopausal status, dose reduction, metastasis diagnosis time, and CDK starting interval (CDK interval). Age, number of metastatic sites, ECOG PS, and menopausal status were found to be significantly associated with PFS ( $p=0.001, p=0.006, p=0.048, p=0.008$, respectively; Table 2$)$. As a result of multivariate analysis, it was determined that taking PPIs was an independent predictor of shortening PFS (hazard ratio 5.60; $95 \%$ confidence interval: 1.98-15.85; $p=<0.001$; Table 2). When we analysed the effective role of PPI use on PFS separately in the letrozole (hormone sensitive) and fulvestrant (hormone resistant) groups, the PFS was significantly shorter in patients using PPIs in both groups ( $p=0.006, p=0.021$ Figure2A, 2B).

In the ribociclib group, the PFS of the patients using PPIs was shorter than the PFS of the patients not using PPIs (12.64 months vs. unreachable, $p=0.003$, respectively; Figure 1B). After a mean follow-up of 15 months, $65 \%$ of the patients who did not take PPIs did not progress. Univariate analysis included age, CDK combination, number of metastatic sites, ECOG, menopausal status, dose reduction, metastasis diagnosis time, and CDK starting interval (CDK interval). No statistical significance was found in any of the univariate analyses. Only PPI use was found to have a significant effect on PFS in patients receiving ribociclib (hazard ratio $2.9 ; 95 \%$ confidence interval: $1.38-6.40 ; p=0.003 ;$ Table 2). When we analysed the effective role of PPI use on PFS separately in the letrozole (hormone sensitive) and fulvestrant (hormone resistant) groups, the PFS was significantly shorter in patients using PPIs in the letrozole group $(p=0.014, p=0.141$ Figure3A, 3B).

In both the palbociclib group and the ribociclib group, there was no statistically significant difference in grade 3-4 adverse events requiring dose reduction between the patient groups taking and not taking PPIs $(p=0.224$, $\mathrm{p}=0.254 ;$ Table 1).

\section{Discussion}

Among different factors such as fast, feeding, concomitant drugs, gastric $\mathrm{pH}$ increase, etc., the $\mathrm{pH}$ solubility of the drug is considered to be the most relevant influencing drug absorption [18]. When stomach pH increases, the effectiveness of oral anticancer drugs with weak base properties decreases due to decreased bioavailability $[3,19]$. To our knowledge, our study was the first to show that concomitant usage of PPIs with palbocicilib/ribociclib in patients with $\mathrm{mBC}$ had a detrimental effect on PFS. We concluded that increasing gastric $\mathrm{pH}$ induced by PPIs may occur through lowering palbociclib plasma concentrations, which affects treatment efficacy and results in shorter progression-free survival. Palbociclib is a weak $\mathrm{pH}$-dependent base with gradually increasing solubility when the $\mathrm{pH}$ rises above 4.5. Rabeprazole-induced changes in post-fed status on palbociclib pharmacokinetics were not considered clinically significant, and no restrictions for concomitant use of PPIs have been reported in palbociclib labelling $[15,20]$. However, the clinical consequences of rabeprazole's ability to reduce efficacy it were not investigated in the study performed by Sun et al. [15]. Additionally, while investigating the effect of rabeprazole on palbociclib pharmacokinetics, giving just 6 days may not have been enough, because in short-term treatment with PPIs, intragastric pH may not be increased throughout the 24-hour interval [13, 21]. In our study, PPIs (mainly pantoprazole, rabeprazole, esomeprazole) were given at no less than half of all palbociclib therapy for a greater and steady rise in intragastric $\mathrm{pH}$.

The PFS of palbociclib with letrozole in paloma 2 and fulvestrant in paloma 3 was 27.6 months and 9.2 months, respectively, and the PFS of ribociclib with letrozole in monalisa 2 and fulvestrant in monalisa 3 was 
25.3 months and 20.5 months, respectively [10-13]. According to our evaluation, the reason why PFS was lower in paloma 3 than in monalisa 3 was that some patients who received 1 step of chemotherapy in paloma 3 were included in the study, while those who received chemotherapy in monalisa 3 were not included in the study. In our study, some of the patients who received letrozole combination or fulvestrant combination had a history of chemotherapy in metastatic disease; therefore, the PFS of our study may be shorter. In the study by Re et al., PFS was 14 months versus 37.9 months in patients who received and did not receive concominant PPIs with palbociclib, respectively. Additionally, no other significant variable affecting PFS was detected in the multivariate analysis [22]. In the results we presented, PFS was similar to that in this trial in patients who received PPIs, but PFS could not be reached yet in patients who did not receive PPIs.

When below the absolute threshold level, although it is not known at this time that the activity of palbociclib may be affected, palbociclib cell potency in vitro (IC50) with free mean steady-state concentration (Css) is comparable with a Css/IC50 ratio of 0.94 [23]. The findings of the present study support the following hypothesis: prolonged treatment with PPIs may reduce palbociclib to plasma levels below the threshold of the minimum effective concentration, thus reducing its effectiveness to some extent. Failure to evaluate the pharmacokinetic changes induced by PPIs in palbociclib is a limitation of our study. Additionally, studies have shown that short-term treatment with rabeprazole reduces fasting palbociclib $\mathrm{Cmax}$ by $80 \%$ and $41 \%$ at fasting and fed, respectively [15].

There is a little evidence in the literature suggesting that agents that alter gastric $\mathrm{pH}$ have no effect on ribociclib absorption $[14,16]$. Samant et al. examined the steady-state pharmacokinetics of ribociclib $(600 \mathrm{mg})$ during PPI use and found no differences in AUC and $\mathrm{C}_{\max }$ between the PPI-using and non-PPI-using groups [14]. However, that is not specified in this study is whether these patients used the drug when they were hungry or when they were full. The different behaviors of ribociclib and palbociclib in acidic media may be due to the difference in their dissolution strength. Consistent with this information, the solubility of ribociclib is $>2.4$ $\mathrm{mg} / \mathrm{ml}$ at pH 4.5 and $0.8 \mathrm{mg} / \mathrm{ml}$ at $\mathrm{pH}>6.8$, while that of palbociclib is $>0.5 \mathrm{mg} / \mathrm{ml}$ at $\mathrm{pH}<4.5$ only $[14,15]$. Examining the in vitro solubility of ribociclib by simulating fasting intestinal fluid $(\mathrm{pH} 6.5)$ and postprandial intestinal fluid ( $\mathrm{pH} \mathrm{5.0)}$ in biorelevant madia, $600 \mathrm{mg}$ was dissolved in $250 \mathrm{ml}$ of fluid [14]. This feature of ribociclib makes it less affected by PPIs, but its absorption may be affected in environments where stomach acid is potently inhibited, especially in fasting conditions. Therefore, it may be more beneficial to take ribociclib with meals in patients taking ribocicline plus PPIs. Saman et al. reported that trough concentration mean ribociclib values $\left(\mathrm{C}_{\text {trough }}\right.$ ) were 597 and $711 \mathrm{ng} / \mathrm{ml}$ in patients with or without PPI at $600 \mathrm{mg}$ dose, respectively [14]. On average, free $C_{s s}$ expressing a broad therapeutic index a reduction in ribociclib $C_{\text {trough, }}$, is unlikely, as it greatly exceeds in vitro cell potency $\left(\mathrm{C}_{\mathrm{ss}} / \mathrm{IC} 50\right.$ ratio $\left.>25\right)$ [23]. But in real life, almost half of the patients use Ribociclib at a dose of $400 \mathrm{mg}$. Therefore, $C_{\text {trough }}$ values may fall below effective levels. With respect to abemaciclib, this drug also shows pharmacokinetic similarities when compared to other CDK4/6 inhibitors. Notable features are saturable absorption with twice daily administration due to smaller volume of distribution and shorter half-life than ribociclib and palbociclib [24].

Whether P-glycoprotein (P-gp) inhibition has an effect on the PFS of PPIs observed in this study is another issue to be answered. According to our knowledge, palbociclib and ribociclib are P-gp substrates and are moderately inhibited by PPIs $[25,26]$. Additionally, tyrosine kinase inhibitor (TKI) pharmacokinetics were found to be altered by pantaprazole through the influence of breast cancer resistance protein (BCRP) and P-gp [21]. If 
the main mechanism of DDI is P-gp had it been inhibited by PPIs, fewer side effects would have been expected in PPI users due to the effect caused by the increase in gastric $\mathrm{pH}$. In the presence or absence of PPIs, as the differences in adverse drug reactions were not statistically significant, so this hypothesis is not compatible with our data. Accordingly, rabeprazole is known to inhibit P-gp activity at appropriate concentrations, and its clinical net effect reduces palbociclib exposure [15]. However, this effect is great at fasting, in environments where the $\mathrm{pH}$ is higher. Therefore, gastric $\mathrm{pH}$ changes due to PPIs appear to be the main mechanism of interaction with drugs that require an acidic microenvironment for dissolution and absorption [27].

Studies to date have reported other instances of DDIs between PPIs and TKIs (i.e. pazopanib, sunitinib, gefitinib, and erlotinib) [7, 28-33]. A meta-analysis of 16 retrospective studies involving various solid tumors with a total of 372418 patients demonstrated that PPI therapy had a significant impact on survival outcomes in patients receiving oral anticancer drugs [34]. The effect of concomitant PPI administration on overall survival and treatment discontinuation, 90 days and 1 year after discontinuation, on overall survival in another 12538 patients retrospective study with solid and haematological tumours evaluated. This study was performed retrospectively in patients treated with TKIs, and PPI use has been shown to be associated with an increased risk of death [35].

There were some limitations of our study. First, the adverse event profile can be underestimated because of the retrospective nature of our study. However, in the current study, dose reductions of CDK inhibitors were performed more than in other clinical trials. We generally used CDK inhibitors in the COVID-19 pandemic because the labelling time of palbociclib and ribociclib by health authorities in our country was May 2020, so physicians are sensitive to dose reduction when grade 3-4 neutropenia develops. Despite these limitations, we collected soluble and reliable data with satisfactory sample sizes. It was clearly demonstrated that concomitant usage of PPIs was associated with shorter PFS. We recommend caution in the long-term use of PPIs in this specific population and the benefits-risks of coadministration of anticancer drugs whose solubility and absorption depend on $\mathrm{pH}$ and strong acid-reducing agents should be evaluated and decided together. If used, PPI selection should be made carefully. For example, rabeprazole may provide more and longer acid suppression than other drugs in the same class; in treatment management H2-antagonists should also be considered instead of PPIs. Increasing the dose of palbociclib in patients using PPIs may theoretically make sense, but in clinical practice it is probably not an effective strategy due to possible off-label effects. If it is necessary to use PPI together with ribociclib, it should be used on a fed.

\section{Declarations}

The authors declare no competing interests.

\section{References}

1. Smelick, G.S., et al., Prevalence of acid-reducing agents (ARA) in cancer populations and ARA drug-drug interaction potential for molecular targeted agents in clinical development. Molecular pharmaceutics, 2013. 10(11): p. 4055-4062.

2. Wedemeyer, R.-S. and H. Blume, Pharmacokinetic Drug Interaction Profiles of Proton Pump Inhibitors: An Update. Drug Safety, 2014. 37(4): p. 201-211. 
3. Budha, N.R., et al., Drug Absorption Interactions Between Oral Targeted Anticancer Agents and PPIs: Is pH-Dependent Solubility the Achilles Heel of Targeted Therapy? Clinical Pharmacology \& Therapeutics, 2012. 92(2): p. 203-213.

4. Mudie, D.M., et al., Quantification of Gastrointestinal Liquid Volumes and Distribution Following a 240 $m L$ Dose of Water in the Fasted State. Molecular Pharmaceutics, 2014. 11(9): p. 3039-3047.

5. Riechelmann, R.P. and M.K. Krzyzanowska, Drug interactions and oncological outcomes: a hidden adversary. ecancermedicalscience, 2019. 13.

6. Hilton, J.F., et al., An evaluation of the possible interaction of gastric acid suppressing medication and the EGFR tyrosine kinase inhibitor erlotinib. Lung Cancer, 2013. 82(1): p. 136-142.

7. Kumarakulasinghe, N.B., et al., EGFR kinase inhibitors and gastric acid suppressants in EGFR-mutant NSCLC: a retrospective database analysis of potential drug interaction. Oncotarget, 2016. 7(51).

8. Vishwanathan, K., et al., The Effect of Food or Omeprazole on the Pharmacokinetics of Osimertinib in Patients With Non-Small-Cell Lung Cancer and in Healthy Volunteers. The Journal of Clinical Pharmacology, 2018. 58(4): p. 474-484.

9. Spring, L.M., et al., Cyclin-dependent kinase 4 and 6 inhibitors for hormone receptor-positive breast cancer: past, present, and future. The Lancet, 2020. 395(10226): p. 817-827.

10. Finn, R.S., et al., Palbociclib and Letrozole in Advanced Breast Cancer. New England Journal of Medicine, 2016. 375(20): p. 1925-1936.

11. Hortobagyi, G.N., et al., Ribociclib as First-Line Therapy for HR-Positive, Advanced Breast Cancer. New England Journal of Medicine, 2016. 375(18): p. 1738-1748.

12. Slamon, D.J., et al., Phase III Randomized Study of Ribociclib and Fulvestrant in Hormone ReceptorPositive, Human Epidermal Growth Factor Receptor 2-Negative Advanced Breast Cancer: MONALEESA-3. J Clin Oncol, 2018. 36(24): p. 2465-2472.

13. Turner, N.C., et al., Overall Survival with Palbociclib and Fulvestrant in Advanced Breast Cancer. New England Journal of Medicine, 2018. 379(20): p. 1926-1936.

14. Samant, T.S., et al., Ribociclib Bioavailability Is Not Affected by Gastric pH Changes or Food Intake: In Silico and Clinical Evaluations. Clinical Pharmacology \& Therapeutics, 2018. 104(2): p. 374-383.

15. Sun, W., et al., Impact of Acid-Reducing Agents on the Pharmacokinetics of Palbociclib, a Weak Base With pH-Dependent Solubility, With Different Food Intake Conditions. Clinical Pharmacology in Drug Development, 2017. 6(6): p. 614-626.

16. Lu, Y., et al., Ribociclib Population Pharmacokinetics and Pharmacokinetic/Pharmacodynamic Analysis of Neutrophils in Cancer Patients. The Journal of Clinical Pharmacology, 2021. 61(8): p. 1054-1068. 
17. Cardoso, F., et al., 4th ESO-ESMO International Consensus Guidelines for Advanced Breast Cancer (ABC 4) $++T h e s e$ guidelines were developed by the European School of Oncology (ESO) and the European Society for Medical Oncology (ESMO). Annals of Oncology, 2018. 29(8): p. 1634-1657.

18. Willemsen, A.E.C.A.B., et al., Effect of food and acid-reducing agents on the absorption of oral targeted therapies in solid tumors. Drug Discovery Today, 2016. 21(6): p. 962-976.

19. van Leeuwen, R.W.F., et al., Drug-drug interactions with tyrosine-kinase inhibitors: a clinical perspective. The Lancet Oncology, 2014. 15(8): p. e315-e326.

20. IBRANCE ${ }^{\circledR}$, Full Prescribing Information. 2016.

21. van Leeuwen, R.W.F., et al., Tyrosine Kinase Inhibitors and Proton Pump Inhibitors: An Evaluation of Treatment Options. Clinical Pharmacokinetics, 2017. 56(7): p. 683-688.

22. Del Re, M., et al., Drug-drug interactions between palbociclib and proton pump inhibitors may significantly affect clinical outcome of metastatic breast cancer patients. ESMO open, 2021. 6(5): p. 100231.

23. Goldstein, M.J., et al., Optimizing the Therapeutic Window of Targeted Drugs in Oncology: PotencyGuided First-in-Human Studies. Clinical and Translational Science, 2021. 14(2): p. 536-543.

24. Braal, C.L., et al., Inhibiting CDK4/6 in Breast Cancer with Palbociclib, Ribociclib, and Abemaciclib: Similarities and Differences. Drugs, 2021. 81(3): p. 317-331.

25. de Gooijer, M.C., et al., P-glycoprotein and breast cancer resistance protein restrict the brain penetration of the CDK4/6 inhibitor palbociclib. Investigational New Drugs, 2015. 33(5): p. 1012-1019.

26. Martínez-Chávez, A., et al., P-glycoprotein limits ribociclib brain exposure and CYP3A4 restricts its oral bioavailability. Molecular pharmaceutics, 2019. 16(9): p. 3842-3852.

27. Ollier, E., et al., In vitro and in vivo evaluation of drug-drug interaction between dabigatran and proton pump inhibitors. Fundamental \& Clinical Pharmacology, 2015. 29(6): p. 604-614.

28. Fang, Y.-H., et al., Concurrent proton-pump inhibitors increase risk of death for lung cancer patients receiving 1 st-line gefitinib treatment-a nationwide population-based study. Cancer management and research, 2019. 11: p. 8539.

29. Ha, V.H., et al., Does gastric acid suppression affect sunitinib efficacy in patients with advanced or metastatic renal cell cancer? Journal of Oncology Pharmacy Practice, 2014. 21(3): p. 194-200.

30. Lalani, A.-K.A., et al., Proton Pump Inhibitors and Survival Outcomes in Patients With Metastatic Renal Cell Carcinoma. Clinical Genitourinary Cancer, 2017. 15(6): p. 724-732.

31. McAlister, R.K., et al., Effect of Concomitant pH-Elevating Medications with Pazopanib on ProgressionFree Survival and Overall Survival in Patients with Metastatic Renal Cell Carcinoma. The Oncologist, 2018. 23(6): p. 686-692. 
32. Veerman, G.D.M., et al., Influence of Cow's Milk and Esomeprazole on the Absorption of Erlotinib: A Randomized, Crossover Pharmacokinetic Study in Lung Cancer Patients. Clinical Pharmacokinetics, 2021. 60(1): p. 69-77.

33. Zenke, Y., et al., Clinical Impact of Gastric Acid-Suppressing Medication Use on the Efficacy of Erlotinib and Gefitinib in Patients With Advanced Non-Small-Cell Lung Cancer Harboring EGFR Mutations. Clinical Lung Cancer, 2016. 17(5): p. 412-418.

34. Indini, A., et al., Impact of Use of Gastric-Acid Suppressants and Oral Anti-Cancer Agents on Survival Outcomes: A Systematic Review and Meta-Analysis. Cancers, 2020. 12(4).

35. Sharma, M., et al., The concomitant use of tyrosine kinase inhibitors and proton pump inhibitors: Prevalence, predictors, and impact on survival and discontinuation of therapy in older adults with cancer. Cancer, 2019. 125(7): p. 1155-1162.

\section{Tables}

Table 1: Clinical characteristics of patients and their distrubution across PPI groups. 


\begin{tabular}{|c|c|c|c|c|c|c|c|c|}
\hline \multirow[t]{3}{*}{ Characteristic } & \multicolumn{4}{|c|}{ PALBOCICLIB } & \multicolumn{4}{|c|}{ RIBOCICLIB } \\
\hline & \multirow{2}{*}{$\begin{array}{l}\text { Total } \\
\text { number } \\
\text { of } \\
\text { patients } \\
(\mathrm{n}=105)\end{array}$} & \multicolumn{2}{|c|}{$\begin{array}{l}\text { Concomitant use of } \\
\text { PPls }\end{array}$} & \multirow[t]{2}{*}{$\begin{array}{l}\mathrm{P} \\
\text { score }\end{array}$} & \multirow{2}{*}{$\begin{array}{l}\text { Total } \\
\text { number } \\
\text { of } \\
\text { patients } \\
(\mathrm{n}=112)\end{array}$} & \multicolumn{2}{|c|}{$\begin{array}{l}\text { Concomitant use of } \\
\text { PPls }\end{array}$} & \multirow[t]{2}{*}{$\begin{array}{l}\mathrm{P} \\
\text { score }\end{array}$} \\
\hline & & No & Yes & & & No & Yes & \\
\hline \multirow{2}{*}{$\begin{array}{l}\text { Age, median } \\
\text { (Range) }\end{array}$} & 59 & 58 & 61 & \multirow[t]{2}{*}{-} & 53 & 49 & 57 & \multirow[t]{2}{*}{ - } \\
\hline & $(32-83)$ & $(35-76)$ & $(32-83)$ & & $(32-87)$ & $(32-87)$ & (38-87) & \\
\hline \multicolumn{9}{|l|}{$\begin{array}{l}\text { Menopausal } \\
\text { status, } n(\%)\end{array}$} \\
\hline \multirow[t]{2}{*}{ Premenopause } & $3<(30.4)$ & $14(30.0)$ & $10(21.1)$ & \multirow{2}{*}{0.514} & $43(38.3)$ & $22(43.1)$ & $21(34.4)$ & \multirow{2}{*}{0.436} \\
\hline & $73(69.5)$ & $26(65.0)$ & $47(72.3)$ & & 69(61.6) & $29(56.9)$ & $40(65.6)$ & \\
\hline \multicolumn{9}{|l|}{ Postmenopause } \\
\hline \multicolumn{9}{|l|}{ ECOG PS, n (\%) } \\
\hline 0 & $19(18)$ & $8(20.0)$ & $11(16.9)$ & \multirow[t]{3}{*}{0.079} & $38(33.9)$ & $23(45.1)$ & $15(24.6)$ & \multirow[t]{3}{*}{0.072} \\
\hline 1 & $74(70.4)$ & $31(77.5)$ & $43(66.2)$ & & $60(53.5)$ & $23(45.1)$ & $37(60.7)$ & \\
\hline 2 & $12(11.4)$ & $1(2.5)$ & 11(16.9) & & $14((12.5)$ & $5(9.8)$ & $9(14.8)$ & \\
\hline \multicolumn{9}{|l|}{$\begin{array}{l}\text { Disease site, } \mathrm{n} \\
(\%)\end{array}$} \\
\hline Viccoral & \multirow{2}{*}{$\begin{array}{l}63(60) \\
42(40)\end{array}$} & $22(55.0)$ & $41(63.1)$ & \multirow[t]{2}{*}{0.421} & $54(48.2)$ & $24(47.1)$ & $30(49.2)$ & \multirow[t]{2}{*}{0.851} \\
\hline Nonvisceral & & $18(45.0)$ & 24(36.9) & & $58(51.7)$ & $27(52.9)$ & $31(50.8)$ & \\
\hline \multicolumn{9}{|l|}{$\begin{array}{l}\text { Endocrine } \\
\text { therapy, n (\%) }\end{array}$} \\
\hline \multirow{2}{*}{$\begin{array}{l}\text { Letrozole } \\
\text { Fulvestrant }\end{array}$} & $49(46.6)$ & $23(57.5)$ & $26(40.0)$ & \multirow[t]{2}{*}{0.107} & $66(58.9)$ & $34(66.7)$ & $32(52.5)$ & \multirow[t]{2}{*}{0.177} \\
\hline & $56(53.3)$ & $17(42.5)$ & $39(60.0)$ & & $46(41)$ & 17(33.3) & $29(47.5)$ & \\
\hline \multicolumn{9}{|l|}{$\begin{array}{l}\text { Dose Reduction, } \\
\text { n (\%) }\end{array}$} \\
\hline \multirow{2}{*}{ Yes } & $56(53.3)$ & 19(47.5) & $37(56.9)$ & \multirow[t]{2}{*}{0.224} & $61(54.4)$ & $25(49.0)$ & $36(59.0)$ & 0.254 \\
\hline & $49(46.7)$ & $21(52.5)$ & $28(43.1)$ & & $51(45.6)$ & $26(51.0)$ & $25(41.0)$ & \\
\hline $\begin{array}{l}\text { CDK inhibitor } \\
\text { interval }\end{array}$ & & & & & & & & \\
\hline$<18$ months & $65(61.9)$ & $27(67.5)$ & $38(58.5)$ & 0.411 & $86(76.7)$ & $41(80.4)$ & $45(73.8)$ & 0.502 \\
\hline$\geq 18$ months & $40(38)$ & 13(32.5) & $27(41.5)$ & & $26(23.2)$ & 10(19.6) & $16(26.2)$ & \\
\hline PPI , n (\%) & & & & & & & & \\
\hline Pantoprazole & & & $28(43.1)$ & & & & $18(29.5)$ & \\
\hline Rabeprazole & & & $17(26.2)$ & & & & $10(16.4)$ & \\
\hline
\end{tabular}


Esomeprazole

Lansoprazole

Omeprazole
8(12.3)

9(13.8)

3(4.6)
$18(29.5)$

$3(4.9)$

12(19.7)

Table 2. Univariate and multivariate analysis for progression-free survival. 


\begin{tabular}{|c|c|c|c|c|c|c|c|c|}
\hline \multirow[t]{3}{*}{ Variables } & \multicolumn{4}{|c|}{ PALBOCICLIB } & \multicolumn{4}{|c|}{ RIBOCICLIB } \\
\hline & \multicolumn{2}{|c|}{ Univariate } & \multicolumn{2}{|c|}{ Multivariate } & \multicolumn{2}{|c|}{ Univariate } & \multicolumn{2}{|c|}{ Multivariate } \\
\hline & $\begin{array}{l}\mathrm{HR} \\
(95 \%) \mathrm{Cl}\end{array}$ & $\begin{array}{l}\mathrm{P} \\
\text { value }\end{array}$ & $\begin{array}{l}\mathrm{HR} \\
(95 \%) \mathrm{Cl}\end{array}$ & $\begin{array}{l}P \\
\text { value }\end{array}$ & $\begin{array}{l}\text { HR } \\
(95 \%) \\
\text { Cl }\end{array}$ & $\begin{array}{l}P \\
\text { value }\end{array}$ & $\begin{array}{l}\mathrm{HR} \\
(95 \%) \\
\mathrm{Cl}\end{array}$ & $\begin{array}{l}\mathrm{P} \\
\text { value }\end{array}$ \\
\hline \multirow[t]{2}{*}{ Age } & 0,94 & 0,001 & 0.95 & 0.053 & 0,99 & 0.758 & & \\
\hline & $\begin{array}{l}(0,91- \\
0,97)\end{array}$ & & $\begin{array}{l}(0.90- \\
1.00)\end{array}$ & & $\begin{array}{l}(0.96- \\
1.02)\end{array}$ & & & \\
\hline \multirow{2}{*}{$\begin{array}{l}\text { Number of } \\
\text { metastatic sites }\end{array}$} & 3,8 & 0.006 & 2.28 & 0.201 & 0.95 & 0.403 & & \\
\hline & $\begin{array}{l}(1.46- \\
10.04)\end{array}$ & & $\begin{array}{l}(0.64- \\
8.09)\end{array}$ & & $\begin{array}{l}(0.56- \\
1.59)\end{array}$ & & & \\
\hline \multirow{2}{*}{$\begin{array}{l}\text { CDK inhibitor } \\
\text { combination }\end{array}$} & 1.8 & 0.089 & & & 1.26 & 0.509 & & \\
\hline & $\begin{array}{l}(0.91- \\
3.67)\end{array}$ & & & & $\begin{array}{l}(0.63- \\
2.48)\end{array}$ & & & \\
\hline \multirow[t]{2}{*}{ ECOG PS } & 0.215 & 0.048 & 0.66 & 0.694 & 0.67 & 0.769 & & \\
\hline & $\begin{array}{l}(0.04- \\
0.98)\end{array}$ & & $\begin{array}{l}(0.09- \\
4.95)\end{array}$ & & $\begin{array}{l}(0.21- \\
2.09)\end{array}$ & & & \\
\hline \multirow[t]{2}{*}{ Pre/Post-menopause } & 0.394 & 0.008 & 0.72 & 0.537 & 0.71 & 0.340 & & \\
\hline & & & $\begin{array}{l}(0.25- \\
2.03)\end{array}$ & & $\begin{array}{l}(0.36- \\
1.41)\end{array}$ & & & \\
\hline \multirow{2}{*}{$\begin{array}{l}\text { Visseral-nonvisseral } \\
\text { disease }\end{array}$} & 0.58 & 0.130 & & & 0.59 & 0.135 & & \\
\hline & $\begin{array}{l}(0,28- \\
1.11)\end{array}$ & & & & $\begin{array}{l}(0.29- \\
1.18)\end{array}$ & & & \\
\hline \multirow[t]{2}{*}{ Dose reduction } & 1.22 & 0.550 & & & 1.21 & 0.587 & & \\
\hline & $\begin{array}{l}(0.62- \\
2.37)\end{array}$ & & & & $\begin{array}{l}(0.60- \\
2.44)\end{array}$ & & & \\
\hline \multirow[t]{2}{*}{ CDK inhibitor interval } & 1.92 & 0.054 & & & 1.41 & 0.361 & & \\
\hline & $\begin{array}{l}(0.99- \\
3.71)\end{array}$ & & & & $\begin{array}{l}(0.68- \\
2.89)\end{array}$ & & & \\
\hline \multirow{2}{*}{$\begin{array}{l}\text { Concomitant use of } \\
\text { PPIs }\end{array}$} & 5.60 & $<0.001$ & 7.85 & $<0.001$ & 2.90 & 0.003 & 2.90 & 0.003 \\
\hline & $\begin{array}{l}(1.98- \\
15.85)\end{array}$ & & $\begin{array}{l}(2.67- \\
23.05)\end{array}$ & & $\begin{array}{l}(1.38- \\
6.40)\end{array}$ & & $\begin{array}{l}(1.38- \\
6.40)\end{array}$ & \\
\hline
\end{tabular}




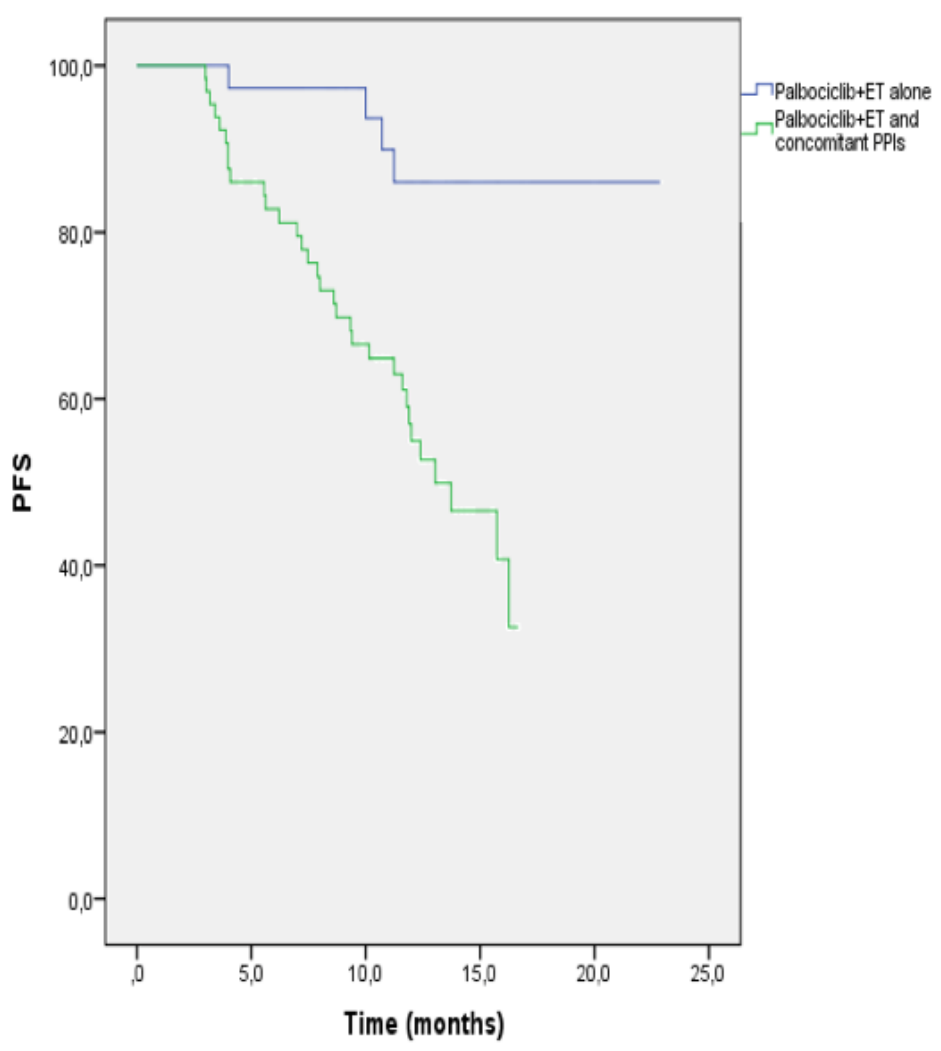

(A)Palhnciclih H HR 7.85

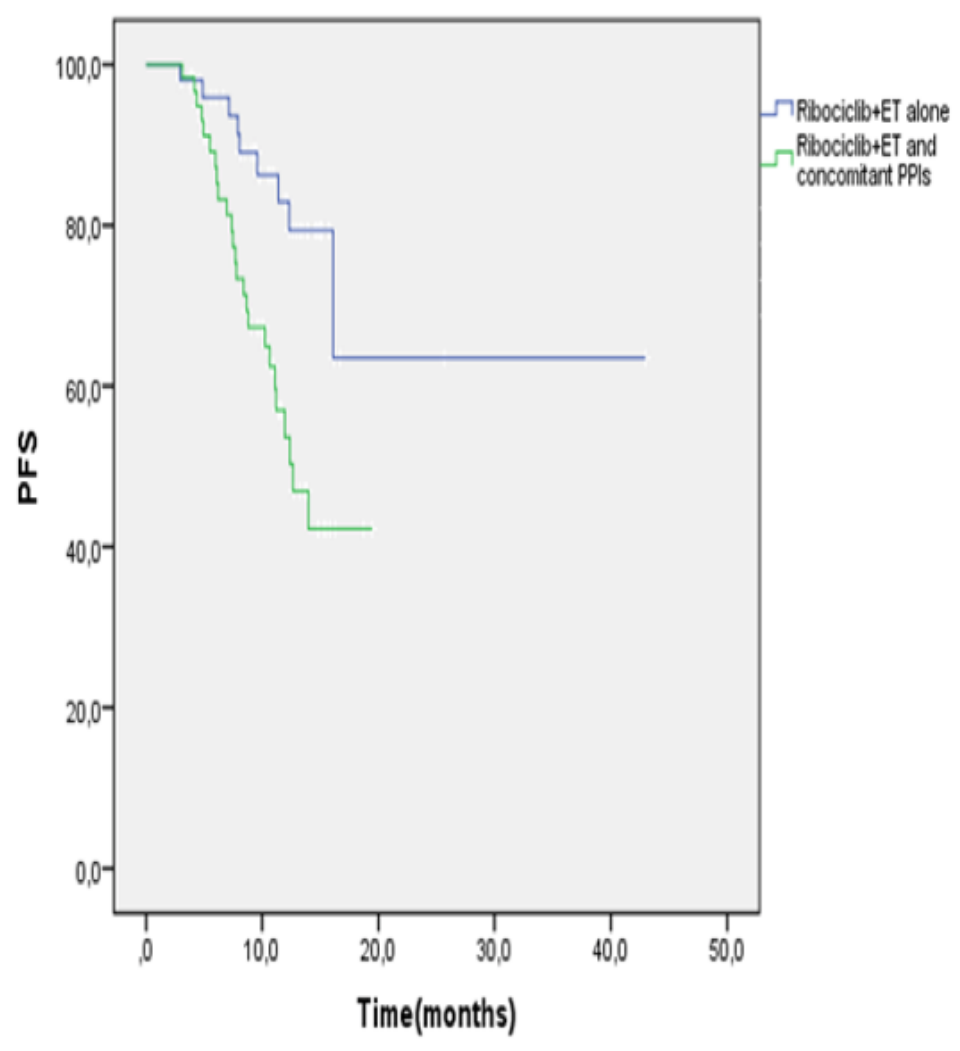

(R) Rihnciclih: HR 2.90

\section{Figure 1}

PFS curves of Palbociclib and Ribociclib combined endocrine therapy with or without PPIs. (Kaplan meier estimates) ET: endocrine treatment, PFS: progression free survival, PPI: proton pomp inhibitör, Cl: confidence interval, HR: hazard ratio 


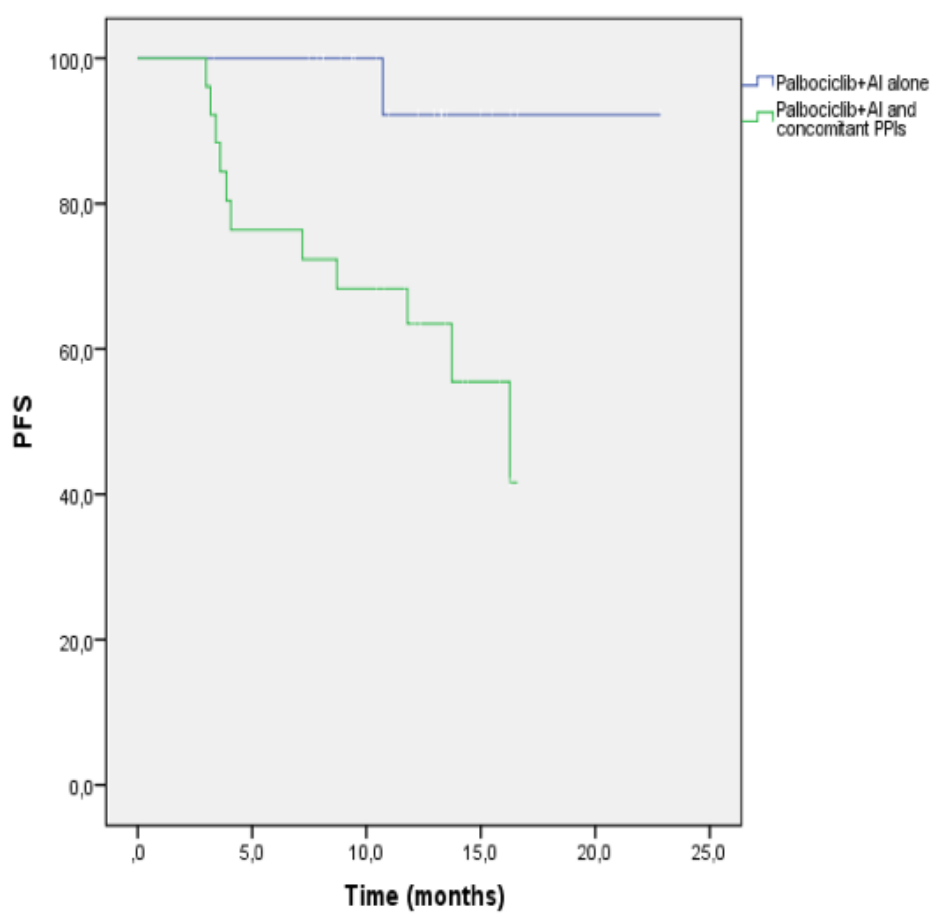

(A)Palbociclib+AI log rank $\mathrm{p}=0.006$

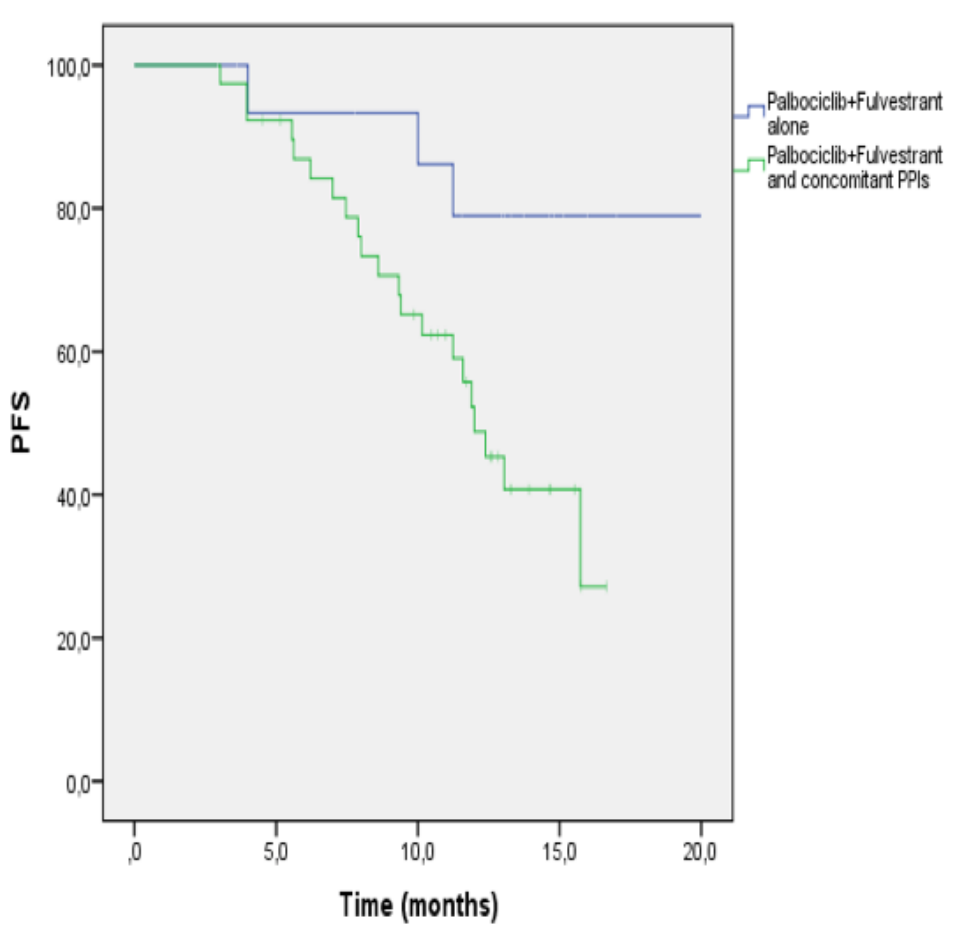

(B)Palbociclib+Fulvestrant $\log$ rank $\mathrm{p}=0.021$

\section{Figure 2}

PFS curves of Palbociclib+Al and Palbociclib+Fulvestrant with or without PPIs. (Kaplan meier estimates) Al: aromatase inhibitor, PFS: progression free survival, PPI: proton pomp inhibitör 


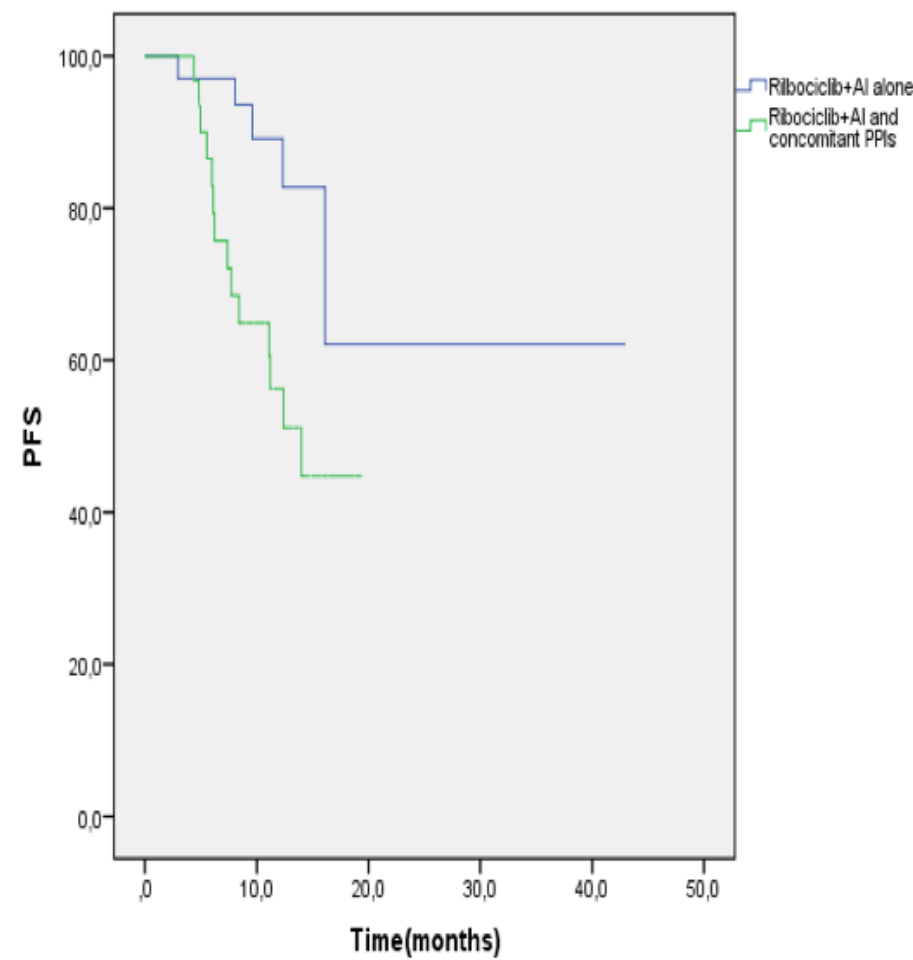

(A)Ribociclib+AI
$\log$ rank $\mathrm{p}=0.014$

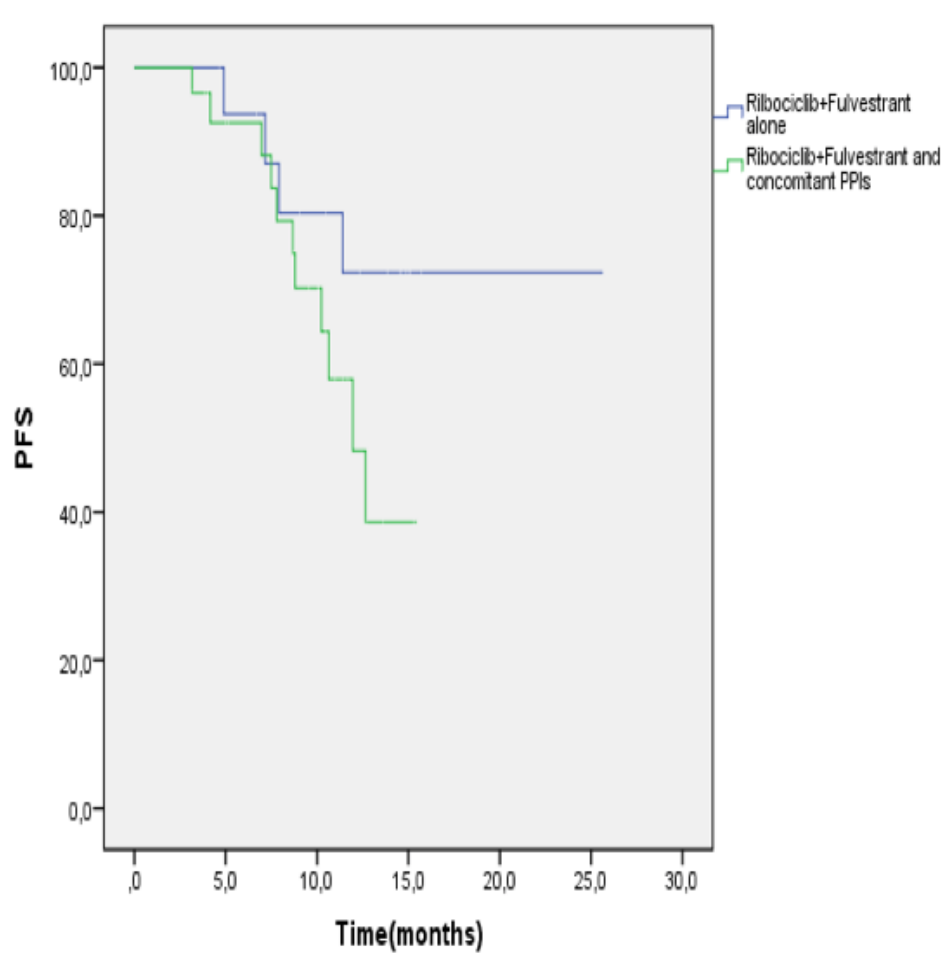

(B)Ribociclib+Fulvestrant $\log$ rank $\mathrm{p}=0.141$

Figure 3

PFS curves of Ribociclib+Al and Ribociclib+Fulvestrant with or without PPIs. (Kaplan meier estimates) Al: aromatase inhibitor, PFS: progression free survival, PPI: proton pomp inhibitör 